\title{
Status of the Regenerative ECLSS Water Recovery System
}

\author{
D. Layne Carter \\ NASA, Marshall Space Flight Center
}

\begin{abstract}
NASA has completed the delivery of the regenerative Water Recovery System (WRS) for the International Space Station (ISS). The major assemblies included in this system are the Water Processor Assembly (WPA) and Urine Processor Assembly (UPA). This paper summarizes the final effort to deliver the hardware to the Kennedy Space Center for launch on STS-126, the onorbit status as of April 2009, and describes some of the technical challenges encountered and lessons learned over the past year.
\end{abstract}

\section{INTRODUCTION}

The U.S. regenerative Environmental Control and Life Support Systems (ECLSS) have been developed to enable the ISS to expand its crew size from three to six crewmembers. Regenerative ECLS includes the Water Recovery System (WRS), comprised of the Urine Processor Assembly (UPA) and Water Processor Assembly (WPA). This hardware produces potable water from a combination of condensate and urine collected on ISS. The Water Recovery System is located in two ISPR racks, named WRS\#1 and WRS\#2. This hardware was delivered to ISS on STS-126 on November 14, 2008. The racks were transferred to and installed in the US Lab D4 and P4 locations, which will be their temporary home until they are relocated to Node 3 in 2010.

\section{WATER RECOVERY SYSTEM}

The layout of the two WRS racks is shown in Figure 1, along with the Oxygen Generation System. The WPA is packaged entirely in WRS rack \#1 and partially in WRS Rack \#2, linked by process water lines running between the two racks. The remaining portion of WRS Rack \#2 houses the UPA.

The following sections provide a description of the WRS, give current status, and describe issues and lessons learned during the past year. For the prior years' status, see references 1-6.
WATER PROCESSOR ASSEMBLY OVERVIEW - A simplified schematic of the WPA is shown in Figure 2.

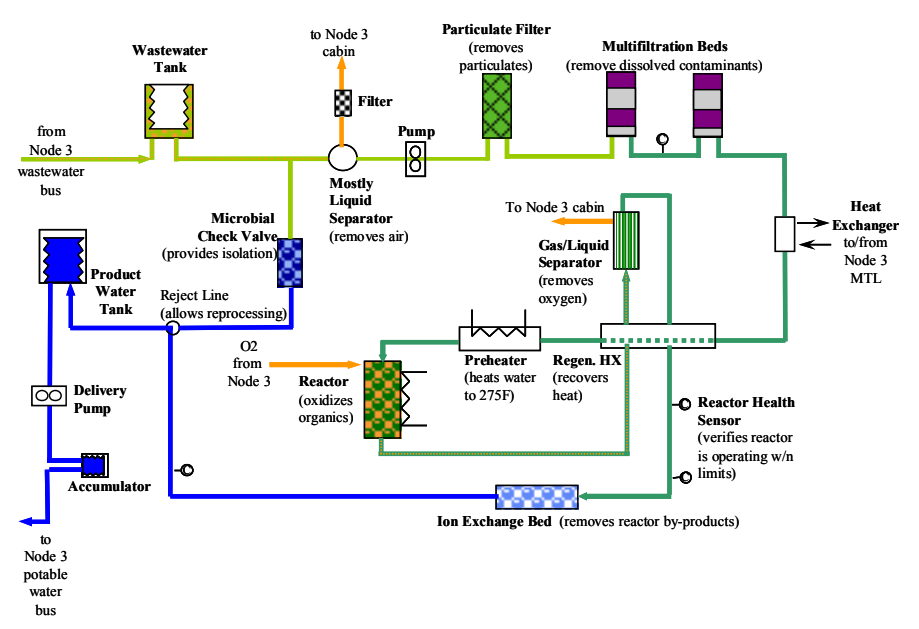

Figure 2. WPA Simplified Schematic

Wastewater delivered to the WPA includes condensate from the Temperature and Humidity Control System and distillate from the UPA. This wastewater is temporarily stored in the Wastewater Tank Orbital Replacement Unit (ORU). Gas is removed from the wastewater by the Mostly Liquid Separator (MLS) (part of the Pump/Separator ORU), and passes through the Separator Filter ORU where odor-causing contaminants are removed from entrained air before returning the air to the cabin. Next, the water is pumped through the Particulate Filter ORU followed by two Multifiltration (MF) Beds where inorganic and non-volatile organic contaminants are removed. Once breakthrough of the first bed is detected, the second bed is relocated into the first bed position, and a new second bed is installed. The Sensor ORU located between the two MF beds helps to determine when the first is saturated based on conductivity. Following the MF Beds, the process water stream enters the Catalytic Reactor ORU, where low molecular weight organics not removed by the filtration process are oxidized in the presence of oxygen, elevated temperature, and a catalyst. A regenerative 


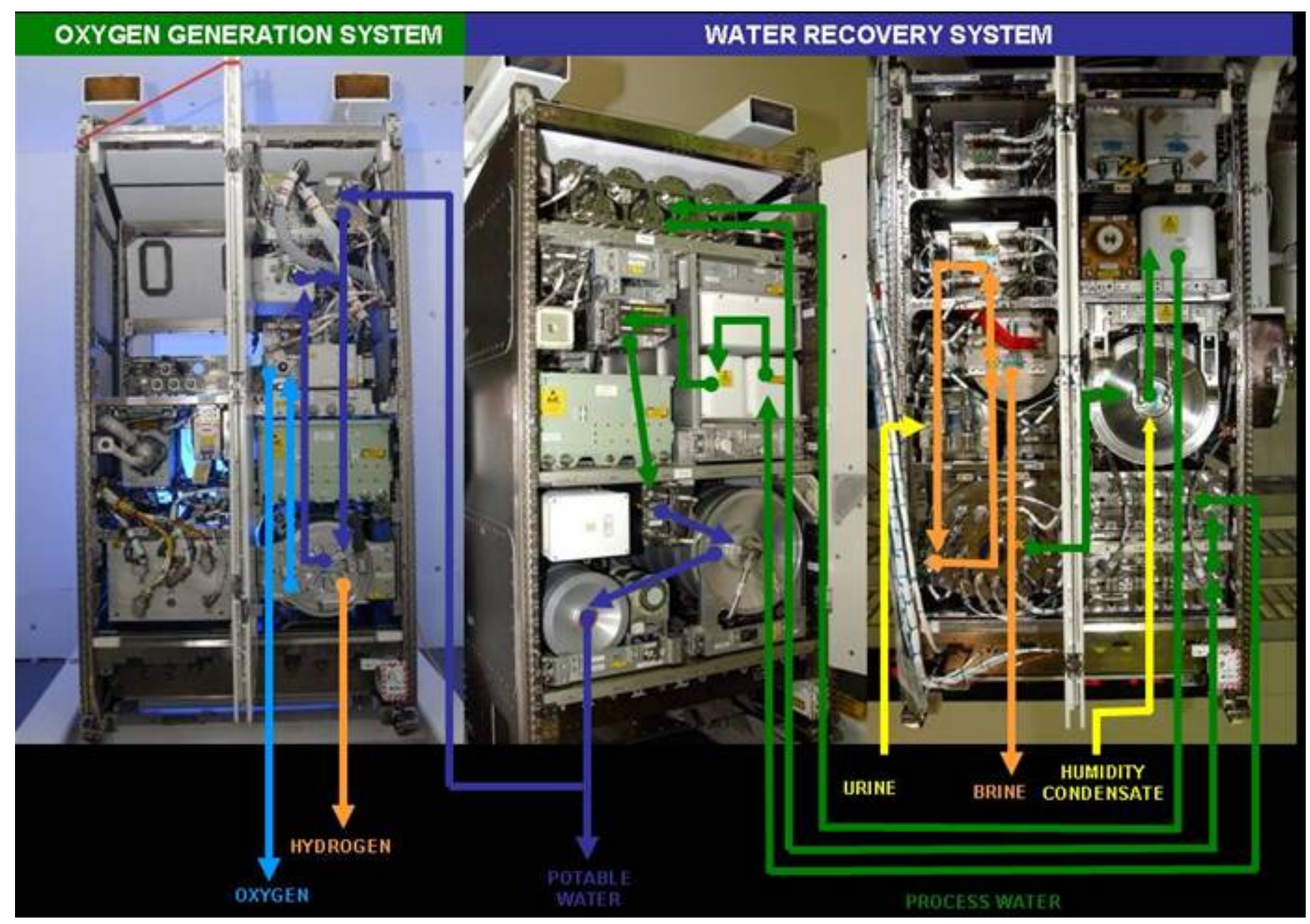

Figure 1. International Space Station Regenerative ECLSS

heat exchanger recovers heat from the catalytic reactor effluent water to make this process more efficient. The Gas Separator ORU removes excess oxygen and gaseous oxidation by-products from the process water and returns it to the cabin. The Reactor Health Sensor ORU monitors the conductivity of the reactor effluent as an indication of whether the organic load coming into the reactor is within the reactor's oxidative capacity. Finally, the Ion Exchange bed ORU removes dissolved products of oxidation and adds iodine for residual microbial control. The water is subsequently stored in the product water tank prior to delivery to the ISS potable water bus. The Water Delivery ORU contains a pump and small accumulator tank to deliver potable water on demand to users. The WPA is controlled by a firmware controller.

URINE PROCESSOR ASSEMBLY OVERVIEW - A simplified schematic of the UPA is shown in Figure 3. Pretreated urine is delivered to the UPA either from the USOS Waste and Hygiene Compartment (outfitted with the Russian urinal) or it can be supplied via manual transfer from the Russian urine container. In either case, the composition of the pretreated urine is the same, including urine, flush water, and a pretreatment formula containing chromium trioxide and sulfuric acid to control microbial growth and control the reaction of urea to ammonia. The urine is temporarily stored in the
Wastewater Storage Tank Assembly (WSTA). The Fluids Control and Pump Assembly (FCPA) is a fourtube peristaltic pump that moves urine from the WSTA into the Distillation Assembly (DA), recycles concentrated waste from the DA into the Recycle Filter Tank Assembly (RFTA), and pumps product water to the wastewater interface with the WPA. The DA is the heart of the UPA, and consists of a rotating centrifuge where the waste urine stream is evaporated at low pressure and condensed on the opposite side of the surface thus conserving latent energy. A rotary lobe compressor provides the driving force for the evaporation and compression of water vapor. Waste brine resulting from the distillation process is stored in the RFTA, which has a capacity of approximately $41 \mathrm{~L}$. The Pressure Control and Pump Assembly (PCPA) is another four-tube peristaltic pump which provides for the removal of noncondensable gases and water vapor from the DA. Liquid cooling of the pump housing promotes condensation, thus reducing the required volumetric capacity of the peristaltic pump. Gases and condensed water are pumped to the Separator Plumbing Assembly (SPA), which recovers and returns water from the purge gases to the product water stream. A Firmware Controller Assembly (FCA) provides the command control, excitation, monitoring, and data downlink for UPA sensors and effectors. 


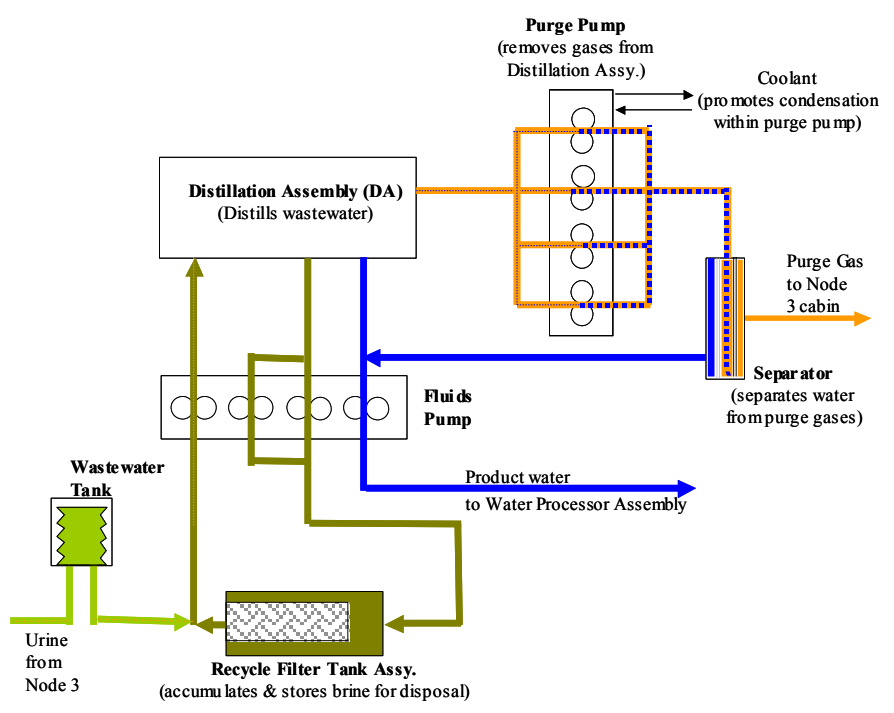

Figure 3. Urine Processor Assembly Schematic

The UPA is designed to process a nominal load of 9 $\mathrm{kg} /$ day (19.8 lbs/day) of wastewater consisting of urine and flush water. This is the equivalent of a 6 -crew load on ISS. Product water from the UPA must meet specification quality requirements for conductivity, $\mathrm{pH}$, ammonia, particles, and total organic carbon. The UPA recovers a minimum of $85 \%$ of the water content from the pretreated urine.

The UPA is packaged into 7 ORUs, which take up slightly more than half of the WRS Rack \#2. The RFTA is the only expendable ORU, sized for a 30-day replacement schedule when processing the daily urine load from 6 crewmembers.

WATER RECOVERY SYSTEM DELIVERY TO ISS Prior to launch, an operational analysis of the Water Processor Assembly identified a potential issue with overpressurization of the WPA Mostly Liquid Separator (MLS). Since the portion of the WPA between the pump (immediately downstream of the MLS) and the Catalytic Reactor ORU operates at elevated pressure (approximately 5 to $6 \mathrm{~atm}$ ), this volume must be depressurized after the WPA completes a processing cycle. During a nominal transition out of Process, a bypass valve is opened around the MLS that allows the system to depressurize to the waste tank. However, if the WPA experiences specific failures during Process mode, this valve will not open and the system will depressurize to the MLS. Since gas may be present in the pressurized portion of the system, the resulting pressure in the MLS could exceed its maximum design pressure (MDP) of $2 \mathrm{~atm}$, resulting in MLS damage. To prevent this scenario from occurring, Hamilton Sundstrand built a check valve that will be located immediately downstream of the pump. This check valve prevents backflow to the MLS and thus precludes the risk of overpressurization. In addition, a software revision is in development at Hamilton Sundstrand that will minimize the risk of damage to the MLS, and will be implemented in 2010.
In addition, an on-orbit dry-out procedure was developed for the Gas Separator ORU. The Gas Separator ORU underwent a major redesign in 2004 to address issues with condensation observed during system testing at Hamilton Sundstrand. Testing conducted with the redesigned configuration indicated that the problem had been corrected. However, in order to mitigate the unknown risks of a) long term operation, b) zero $G$ effects, and c) unforeseen contingency scenarios, an onorbit dry-out procedure has been developed. This would allow the ORU to be recovered on-orbit in the event that performance becomes degraded or lost due to condensation in the sweep gas circuit of the ORU. Testing to validate the dry-out procedure was successfully conducted at Hamilton Sundstrand in 2007. However the testing showed that condensate could build up in sweep gas lines and effectively block sweep gas flow to entire sections of the ORU. A GS dry-out kit was designed, built, and delivered by MSFC that could be used with the Russian air compressor to blow cabin air through the Gas Separator sweep plumbing and therefore remove any water that was blocking the sweep air flow. This hardware is currently on ISS and will be used if required to recover GS performance.

The WRS Racks were installed in the US Laboratory Module on November 18, 2008. Since the Distillation Assembly, the Catalytic Reactor, and the Reactor Health Sensor ORUs were shipped separate from the racks, these ORUs were next installed in their respective racks. The UPA FCPA and PCPA were transitioned from their hard mounts (for launch loads) to the soft mounts designed to minimize vibrational and acoustic loads during operation. Finally, the MLS check valve discussed previously was installed. Once the racks were fully integrated, the WPA was powered and a flush of the Catalytic Reactor was performed. The Catalytic Reactor is packed with a catalyst that generates fines due to launch loads. These fines are detrimental to the performance of the downstream Gas Separator, therefore a reactor flush is performed after launch of the reactor to minimize the quantity of fines that reach the Gas Separator.

As part of the initial operating procedure on ISS, the coolant flow rate to WRS\#2 was measured. The WRS\#2 Interface Control Document specifies a minimum coolant flow rate of $45.4 \mathrm{~kg} / \mathrm{hr}(100 \mathrm{lb} / \mathrm{hr})$. However, the ISS Moderate Temperate Loop (MTL) in the US Laboratory has flow rate limitations, and was unable to verify a minimum flow rate of $45.4 \mathrm{~kg} / \mathrm{hr}$. Though this value was nominal achieved, fluctuations in the flow rate and sensor error prevent verification of the specified flow rate. Since only UPA hardware receives coolant in WRS\#2, the UPA operation was initiated with additional monitoring of the UPA system performance to detect any adverse effects associated with the reduced coolant flow rate. An engineering review of the on-orbit data has concluded that there has been no impact on UPA performance due to the reduced coolant flow rate. 


\section{URINE PROCESSOR ASSEMBLY CURRENT STATUS}

- The UPA was initially activated on November 20, 2008, on Flight Day 7 of ULF2. The WSTA was launched with $9.1 \mathrm{~L}(20 \mathrm{lb})$ of deionized water (pretreated with oxone and sulfuric acid for microbial control). To initiate UPA operations, this fluid was transferred to the RFTA followed by an additional $60 \mathrm{lb}$ of pretreated urine transferred from the Russian segment in an EDV (urine collection bag developed by the Russian Space Agency). This established the configuration of the UPA required for initiating the urine processing sequence.

Though the first process cycle of the UPA was successful, the UPA experienced a fault on November $21^{\text {st }}$ due to anomalous centrifuge speed and coinciding with high DA motor current. The centrifuge has three speed sensors, which the software uses to define an average speed (by calculating the average of the two sensors closest in value). The S41 sensor was reporting erratic values while the other two sensors were stable, indicating the centrifuge was actually contacting the $\$ 41$ sensor located on the front plate. To address the possibility that the soft mount attachment was allowing the DA to vibrate excessively, the crew was instructed to remount the DA on the hard mounts used to launch the ORU. After this change, the DA periodically exhibited a similar signature in terms of DA speed and motor current, but never to the point that a fault occurred. After several days of this intermittent behavior, the DA performance returned to nominal and the anomaly did not recur.

The UPA processed $180 \mathrm{~kg}$ (400 lb) of pretreated urine over the course of the next six weeks, achieving $81 \%$ recovery of the feed water. On December 28, 2008, the UPA experienced a second failure, this time due to high motor current on the Distillation Assembly. Though similar to the first failure, the anomalous centrifuge speed observed previously was not present during this second failure. Multiple attempts to restart the UPA were unsuccessful, and the DA was replaced with Serial Number 02 (S/N 02) during the 15A mission on March 20, 2009. DA S/N 02 was installed on the hard mounts from the beginning, and had processed $90 \mathrm{~kg}(200 \mathrm{lb})$ of pretreated urine in this configuration as of April 23 with no indication of the underspeed or low motor current observed with DA S/N 01 . DA S/N 01 was returned to MSFC for investigation, which is currently ongoing. This investigation is incomplete at the time this paper is released, but initial observations include that the centrifuge was in fact contacting two of the three speed sensors, and that the compressor lobes were the source of the second failure due to mechanical contact with the compressor endplate.

A second anomaly occurred during the $15 \mathrm{~A}$ mission when preparing for operation with DA S/N 02 . The second RFTA (S/N 03) was installed because the first RFTA (S/N 01) was nearly expended, and it was necessary to return $\mathrm{S} / \mathrm{N} 01$ at the completion of the $15 \mathrm{~A}$ mission to support the refurbishment schedule for future use on ISS. The RFTA fill is accomplished using a vacuum/backfill procedure. The RFTAs are pulled down to a vacuum of less than 200 mTorr prior to launch. Once on ISS, the WSTA volume (operating at 1 to 1.1 atm, or 0 to 1 psig) is opened to the RFTA (still at vacuum), and the pretreated urine is transferred by pressure drop. However, the fill of RFTA S/N 03 was unsuccessful, resulting in the transfer of only $20 \mathrm{~kg} \mathrm{(44}$ lb), significantly less than the RFTA capacity of $41 \mathrm{~kg}(90$ $\mathrm{lb})$ and the minimal requirement of $35 \mathrm{~kg}(78 \mathrm{lb})$. The RFTA S/N 03 was replaced with S/N 02, which was filled with only $32 \mathrm{~kg}(70 \mathrm{lb})$ of pretreated urine. Though it didn't meet the minimal requirement of $35.4 \mathrm{~kg}$, UPA engineering determined that the system could be operated with this transfer quantity, though only $213 \mathrm{~kg}$ $(470 \mathrm{lb})$ of urine would be processed (instead of $231 \mathrm{~kg}$ as planned for a full RFTA). A failure investigation has focused on the two RFTA filters. A pleated polypropylene 10 micron depth filter is located at the effluent of the RFTA to remove any precipitants that may form in the RFTA brine prior to delivery to the DA. In addition, a 100 micron metal cone filter is immediately downstream of the 10 micron filter, with the function of removing any particulates associated with degradation of the 10 micron filter after extended exposure to the urine brine. When filling the RFTA, the flow actually enters the RFTA effluent to take advantage of the nominal fluid lines and associated valving. The failure investigation indicates that the reason for the inadequate fill is due to either particulate loading of the RFTA filters, or due to fluid dynamics associated with the pressure drop through the RFTA filters in microgravity. Ground tests have shown that pretreated urine foams significantly when exposed to vacuum, though this did not prevent fluid transfer when evaluated with flight filters during ground tests. To attempt to utilize the RFTA S/N 03 on ISS, an alternate method has been developed to complete the RFTA fill. This method will require the crew to connect the fill hose to the RFTA inlet, which will bypass the filters. This method will verify the reason for the failure is due to the filters, and allow the RFTA S/N 03 to be fully utilized prior to return to the ground for refurbishment.

After processing $90 \mathrm{~kg}(200 \mathrm{lb})$ of pretreated urine after installation of DA S/N 02, the UPA experienced another failure associated with the FCPA check valve. The purpose of this check valve is to prevent backflow from the WPA waste tank into the DA. This check valve exhibited indications of sticking during initial operations on ISS. The valve would stick during Standby mode, and would result in a high pressure fault or warning when the fluids pump initiated flow. However, the pressure spike associated with the pump operation was always sufficient to open the check valve and thereby allow the UPA to begin operation upon restart. However, on April 23 the check valve failed closed during normal operation of the fluids pump, and repeated attempts to open it by operating the pump were unsuccessful. Since this check valve is a redundant control against backflow (and not required as a safety control), it has been removed from subsequent builds of 
the FCPA. Furthermore, it will be removed from the onorbit FCPA following the development of an on-orbit procedure and provision of required tools.

\section{WATER PROCESSOR ASSEMBLY CURRENT} STATUS - The WPA was initially activated on November 22, 2008. As of May 12, the WPA has processed approximately $270 \mathrm{~kg}(600 \mathrm{lb})$ of urine distillate and $340 \mathrm{~kg}(740 \mathrm{lb})$ of condensate to potable water. Table 1 summarizes the WPA water quality based on chemical analysis of samples returned to the ground. Additional on-orbit analyses are performed by the Total Organic Carbon Analyzer (TOCA), but these values are consistent with the ground analyses and will not be presented in this paper. The initial sample on ISS showed several parameters at elevated levels (though only nickel was actually out of specification) due to the 7 months of storage between final testing at MSFC and operation on ISS. These parameters returned to nominal levels after a few WPA process cycles. The onorbit Water Microbial Kit (WMK) was used to assess the microbial count delivered by the WPA. Microbial counts have been consistently non-detectable, with the exception of the on-orbit count on January 022009 (7 $\mathrm{CFU} / \mathrm{ml}$ ) that is suspected to be due to contamination of the sampling hose. Subsequent microbial analyses at this location have been reported as non-detectable. Based on this data, approval for crew consumption of WPA product water was given on May 1, 2009.

Performance of the WPA on ISS has been successful, with only one minor anomaly. The liquid sensor in the Gas Separator (GS) triggered a fault while the WPA was in standby after the first operating cycle. This liquid sensor is located at the point where the GS sweep air is plumbing into the Avionics Air Assembly (AAA) duct in WRS\#1. After reviewing the GS pressure data, it was concluded that the GS lumens had not ruptured based on the fact that the GS maintained pressure during flow, and that the pressure decreased when flow was ceased at the same rate exhibited during ground tests. Based on this observation, it was determined that the liquid sensor was detecting condensation in the GS sweep plumbing that had migrated to the liquid sensor. This was not an unexpected result, since ground testing at Hamilton Sundstrand had shown that the GS could possibly condensate water vapor in the sweep plumbing during transitional phases. To continue WPA operations, this sensor was inhibited. Trend analysis of the liquid sensor has shown that condensation is detected on the sensor for up to five days following a process cycle.

In addition to the on-orbit activities, WPA engineers have been pursuing a modification to address a concern with the on-orbit design of the gear pumps delivered by Hamilton Sundstrand, including the process pump and delivery pump for the WPA and the recirculation pump for the OGS. These pumps have operated nominally on ISS, though ground processing has shown a tendency for the pump gears to lock up due to dehydration of particles from the pump gears (alumina) resulted in the formation of alumina hydroxide. The alumina hydroxide forms a hard structure that can prevent gear rotation with the limited force available from the flight motor. For the existing pump design, ground handling procedures are in place to insure the gears are always maintained wet, thus minimizing the possibility of dehydration. A pump redesign is also in work that would replace the alumina gears with Stellite gears, while also addressing cycle life through the implementation of a trough in the wear surface to address gear wear during pump startup.

Based on ground tests, the WRS consumes a timeaveraged $743 \mathrm{~W}$-hr/hr while processing wastewater (including $320 \mathrm{~W}-\mathrm{hr} / \mathrm{hr}$ by the WPA, $315 \mathrm{~W}-\mathrm{hr} / \mathrm{hr}$ by the UPA, $94 \mathrm{~W}$-hr/hr by the Avionics Air Assembly (AAA), and a maximum of $14 \mathrm{~W}-\mathrm{hr} / \mathrm{hr}$ dissipated by the Remote Power Control Module (RPCM)) and $297 \mathrm{~W}-\mathrm{hr} / \mathrm{hr}$ while in standby (including $133 \mathrm{~W}-\mathrm{hr} / \mathrm{hr}$ by the WPA, $56 \mathrm{~W}$ $\mathrm{hr} / \mathrm{hr}$ by the UPA, $94 \mathrm{~W}-\mathrm{hr} / \mathrm{hr}$ by the AAA, and a maximum of $14 \mathrm{~W}$-hr/hr by the RPCM).

\section{SUMMARY}

In 2008, the ISS WRS Racks were successfully delivered and operated on ISS. Operation of the Water Processor Assembly has been successful, culminating in the approval of water for crew consumption in April 2009. The Urine Processor Assembly has two issues that must be overcome to declare it fully operational, including the failure of the Distillation Assembly and the failure of the RFTA fill. Resolution of these issues is ongoing and will be documented in a subsequent paper.

Table 1. Summary of WPA Water Quality Data

\begin{tabular}{|l|l|l|l|l|l|l|l|l|l|}
\hline Parameter & $11 / 22 / 08^{*}$ & $11 / 25 / 08$ & $11 / 26 / 08$ & $11 / 26 / 08$ & $12 / 08 / 08$ & $02 / 09 / 09$ & $02 / 27 / 09$ & $03 / 10 / 09$ & $03 / 25 / 09$ \\
\hline $\begin{array}{l}\text { Total Organic } \\
\text { Carbon }(\mathrm{mg} / \mathrm{L})\end{array}$ & 1.05 & 0.35 & 0.23 & 0.19 & 0.23 & 0.12 & 0.12 & 0.09 & 0.09 \\
\hline Ethanol $(\mathrm{mg} / \mathrm{L})$ & $<0.1$ & $<0.1$ & $<0.1$ & $<0.1$ & $<0.1$ & $<0.1$ & $<0.1$ & $<0.1$ & $<0.1$ \\
\hline Acetone $(\mathrm{mg} / \mathrm{L})$ & 0.174 & 0.031 & 0.011 & 0.009 & 0.016 & $<0.002$ & $<0.002$ & $<0.002$ & $<0.002$ \\
\hline $\begin{array}{l}\text { Conductivity } \\
(\mu \mathrm{mhos} / \mathrm{cm})\end{array}$ & 6 & 3 & 3 & 3 & 9 & 3 & 3 & 3 & 3 \\
\hline Nickel $(\mathrm{mg} / \mathrm{L})$ & 1.69 & 0.42 & 0.05 & 0.13 & 0.10 & 0.11 & 0.25 & 0.12 & 0.04 \\
\hline $\mathrm{pH}$ & 7.7 & 7.48 & 7.06 & 7.43 & 7.79 & 6.91 & 6.82 & 6.49 & 6.03 \\
\hline lodine $(\mathrm{mg} / \mathrm{L})$ & 0.21 & 1.40 & 1.89 & 1.90 & 2.41 & 2.54 & 2.70 & 2.70 & 2.71 \\
\hline
\end{tabular}

*represents primarily residual water in the WPA at launch, not processed water 


\section{REFERENCES}

1. Bagdigian, R.M., D.L. Carter, and G. Sitler, "Status of the Regenerative ECLSS Water Recovery System", SAE 2008-01-2133, presented at the $38^{\text {th }}$ International Conference on Environmental Systems, San Francisco, California, July, 2008.

2. Bagdigian, R. M., D. Cloud, J. Bedard, "Status of the Regenerative ECLSS Water Recovery and Oxygen Generation Systems", SAE 2006-01-2057, $36^{\text {th }}$ International Conference on Environmental Systems, Norfolk, Virginia, July, 2006.

3. Bagdigian, R. M., and D. Cloud, "Status of the International Space Station Regenerative Water Recovery and Oxygen Generation Systems", SAE 2005-01-2779, $35^{\text {th }}$ International Conference on Environmental Systems, Rome, July 2005

4. Link, D.E., "Integration of Regenerative ECLSS Functions into the International Space Station U.S. Laboratory Element", SAE 2005-01-2780, $35^{\text {th }}$ International Conference on Environmental Systems, Rome Italy, July 2005.

5. Carrasquillo, R.L., Cloud, D., and Kundrotas, R., "Status of the Node 3 Regenerative ECLSS Water Recovery and Oxygen Generation Systems," SAE 2004-01-2385, $34^{\text {th }}$ International Conference on Environmental Systems, July 2004.

6. Bagdigian, R. M., D. Layne Carter, and D. Cloud, "Status of the International Space Station Regenerative Water Recovery System", SAE 2007-01-3000, $37^{\text {th }}$ International Conference on Environmental Systems, Chicago, IL.

\section{CONTACTS}

Donald Layne Carter

NASA Marshall Space Flight Center

layne.carter@nasa.gov 